\title{
Proceedings of the meeting of the Association of British Neurologists held jointly with the American Neurological Association, in London, 1-2 November 1985
}

CONTROLLED TRIAL OF THYROTROPIN RELEASING HORMONE IN MOTOR NEURONE DISEASE

MH Brooke, J Florence, SL Heller, KK Kaiser. Washington University School of Medicine, St Louis, Missouri, USA

Thyrotropin releasing hormone (T.R.H.) has been reported to cause dramatic improvement in motor neurone disease. We undertook a double-blind controlled trial of the drug in 30 patients. The drug/placebo was administered for two months followed by a two month "wash-out". Evaluation of strength, functional ability, respiratory function, and EMG was included in the protocol. Patients were randomised in strata depending on whether they were ambulatory or non ambulatory, male or female. The average muscle strength of both groups declined significantly during the trial period. A definite increase in the strength of some muscles was detected immediately following the administration of TRH $(p<0.05)$. No change in functional performance was noted. Analysis of the electromyogram showed that the compound action potential was decreased $(p=0.02)$. In spite of the change in the strength, neither the patients nor the investigators believed the effects were of any clinical significance.

MEMBRANE ALTERATIONS IN DUCHENNE DYSTROPHY: THE ROLE OF $\mathrm{Ca}^{2}+$ ACTIVATED NEUTRAL PROTEASE

B Nagy, FJ Samaha. University of Cincinnati College of Medicine, Cincinnati, Ohio, USA

It is known that the sarcoplasmic reticulum (SR) of Duchenne dystrophic muscle showed abnormal calcium uptake and ATPase activity. Our recent studies have shown that normal human muscle SR yields three major protein bands of 100,55 and 45 kilo dalton mass respectively. In 16 normals these proteins have a relative distribution of $55.4,14.6$ and $30 \%$; similar studies on 15 Duchenne dystrophic patients showed an altered protein distribution of decreased 100 kilo dalton mass protein band, while the 55 and 45 kilo dalton mass bands were increased. Disease control studies in seven patients with polymyositis and seven patients with myotonic dystrophy showed a normal distribution of proteins. Protease inhibitors pepstatin, antipain and leupeptin as well as EGTA or EDTA significantly reduced this change implying the presence of a $\mathrm{Ca}^{2}+$ activated protease. Further studies revealed a 5 to 10 fold increase of this enzyme activity in Duchenne dystrophic muscles.

We partially purified the protease which degraded SR in a manner described above in Duchenne dystrophic muscle. Further preliminary studies indicate similar membrane protein degradation in the sarcolemma. These new observations along with those implicating the $\mathrm{Ca}^{2}+$ activated neutral protease in other alterations in Duchenne muscle provide support for a hypothesis on the cause of Duchenne dystrophy. Either as a primary or secondary alteration, high $\mathrm{Ca}^{2}+$ activated neutral protease activity may be the abnormality that leads to the death of the Duchenne dystrophic muscle cell.

CENTRAL MOTOR CONDUCTION IS ABNORMAL IN MOTOR NEURON DISEASE

DA Ingram, M Swash. The London Hospital, Whitechapel, London

Conduction in the central pathways of the brain and spinal cord was studied in 12 patients with motor neuron disease, using transcutaneous electrical stimulation of the cortex, cervical cord (Cv6) and conus medullaris (Lvl) to determine motor latencies to the biceps brachii, thenar eminence and tibialis anterior (TA) muscles. Thoracic cord stimulation (Tv8) was also employed when responses to $\mathrm{Cv} 6$ stimulation were absent. Spinal motor conduction velocity was calculated as previously described (Swash and Snooks, 1984). Central motor conduction time (cortex-Cv6) was normal in all cases in which this could be calculated. Spinal motor conduction velocities ranged from 9 to $74 \mathrm{~m} / \mathrm{s}$ (normal mean $\pm \mathrm{SD}, 59.5 \pm$ $11.3 \mathrm{~m} / \mathrm{s})$. Abnormalities of spinal conduction were often asymmetrical and correlated with the clinical features of corticospinal in volvement. In 2 patients the motor responses in TA were dispersed following Cv6 stimu- $\stackrel{\overrightarrow{+}}{+}$ lation. In general it proved more difficult too excite central motor pathways in patients than in normal controls. The results imply that the functional motor deficit in the $\mathrm{CNS}_{\overline{\mathbb{D}}}^{-}$ in motor neuron disease is of greater clinical importance than has been realised, although often masked by the lower motor neuronfeatures.

\section{Reference}

Swash M, Snooks SJ. Motor conduction velocity in the human spinal cord. J Physiol (Lond) 1984, 360:50P THE NEUROLOGICAL PRACTICE OF A DISTRICT
GENERAL HOSPITAL

J Morrow, V Patterson. Royal Victoria HEspital, Belfast

Downe Hospital is a two-physican hosp without a visiting neurologist, 22 miles fromos Belfast, which serves a catchment population of 53,000. We carried out a prospective study of all medical admissions between August 1983 and July 1984 to determine the frequency of admissions with neurologicab problems and to study how these problems were managed. There were 925 medical admissions of whom $180(19.5 \%)$ had a pri $\overrightarrow{\bar{O}}$ mary neurological cause and a further 193 $(2.1 \%)$ in whom a neurological disorder con-? tributed to admission. Cerebrovascular dis=0 eases accounted for $33.8 \%$ of neurologica cases with seizure disorders $(20 \cdot 5 \%)$, the second commonest group. The neurological patients were investigated as follows: EEG $31 ;$ lumbar puncture 29; isotope brain scan 26 CT brain scan 2; digital subtraction angiography 2 . Only 15 of the neurological patients $(8 \cdot 3 \%)$ were referred for a specialisto neurological or neurosurgical opinion. This study demonstrates firstly that neurologicat disease is common in district general hospitals, secondly that most such patients never see a neurologist and thirdly that their neu= rological investigations may be inap- $N$ propriate. It should be possible to improve the management of such patients by in- 
creasing neurological expertise in the district general hospital. The precise way to do this is not yet clear.

RESIDUAL VISUAL FUNCTION IN SUBJECTS WITH LESIONS OF THE RETROCHIASMAL VISUAL PATHWAYS

IM Blythe, C Kennard, * KH Ruddock. Department of Physics (Biophysics), Imperial College, London. *Department of Neurology, The London Hospital, Whitechapel, London

There has recently been considerable interest in the concept of "blind-sight", 1 in which patients with lesions of the retrochiasmal visual pathways show visually guided behaviour to stimuli located in their visual scotoma (as assessed by standard perimetry). Since this has largely consisted of case reports or small series we have extensively studied a group of 25 patients with such lesions resulting in complete homonymous quadrant or hemianopia. Tests of residual visual function included localisation by saccadic eye movements and pointing, pattern recognition and movement perception. Twenty of the subjects were unable to perform the tests better than chance when stimuli were located within the scotoma. In contrast the remaining five subjects were able to perform accurate eye movements in response to targets flashed within the "blind" field, and detect fast but not slow moving targets. We show that the pathways which give rise to these residual functions mediate virtually normal spatial resolution for target localisation, but provide no discrimination of spatial pattern. This functional differentiation between the location of the target and discrimination of its shape is consistent with the "two visual systems": retinogeniculo-striate and retino-collicularinterpretation of visual organisation. ${ }^{2}$

\section{References}

${ }^{1}$ Schneider GE, Science, (1969) 163; 895-902

${ }^{2}$ Weiskrantz L et al., Brain, (1974) 97; 709-728

READINESS POTENTIAL OF CORTICAL AREA 6 IN PARKINSON'S DISEASE. EVIDENCE FOR A DOPAMINERGIC STRIATAL CONTROL OF POSTURAL SET INVOLVING SUPPLEMENTARY MOTOR AREA

JA Simpson, AJ Khuraibet. Glasgow University Department of Neurology, Institute of Neurological Sciences, Glasgow
Initiation of voluntary movement in Parkinsonism is not correlated with a prolonged Readiness Potential (RP: Bereitschaftspotential) of pre-central cortex. In $96 \%$ of patients with Parkinson's disease it is short ( $<800 \mathrm{~ms}$ ) low amplitude, absent, or positive going. RP is less negative or more positive in pre-adolescence and old age. The negative variation is reduced in duration and amplitude proportionately to severity of Parkinsonism, but varies in parallel with short term fluctuations of akinesia suggesting dopaminergic striatal control but no disconnection.

Abnormal RP does not correlate with slow reaction time; movement time, or power. Abnormality is closely associated with defective anticipatory postural responses. Lower limb postural responses occur after the (upper limb) prime mover, with bilateral proximal muscles following calf muscles instead of preceding them (like normal subjects on an unstable platform).

Our findings support a model of the basal ganglia as a gate-control for postural set with an efferent pathway involving the premotor and supplementary motor areas, and possibly bilateral cortico-rubro-spinal pathways for body-on-body and head-on-body adjustments. The negative RP reflects activity there and is not a marker of the central processing involving the decision making and the command of area 4

\section{DOPAMINE METABOLISM IN HUMAN BRAIN} MEASURED IN VIVO WITH PET

KL Leenders. MRC Cyclotron Unit, Hammersmith Hospital, London

The fate of trace amounts of dopaminergic compounds, labelled with short-lived positron emitting isotopes like ${ }^{18} \mathrm{~F}$ or ${ }^{11} \mathrm{C}$, can be followed in vivo in human brain using positron emission tomography (PET). L- $\left({ }^{18} \mathrm{~F}\right)$ fluorodopa, an analogue of levodopa, was administered intravenously in a group of healthy volunteers $(n=10)$ and in patients with Parkinson's disease (PD; $\mathrm{n}=20$ ), Huntington's chorea ( $\mathrm{HC} ; \mathrm{n}=1)$, Progressive Supranuclear Palsy (PSP; $\mathbf{n}=5$ ) and hemidystonia (HD; $n=6$ ). A series of consecutive 10 minute scans at the level of the striatum was then performed using the single slice ECAT-II tomograph.

Most of the activity was concentrated in the striatal regions and decreased only slightly over time in controls. The activity in surrounding brain diminished more quickly than in the striatum. The ratio of activity between striatum and surrounding brain in- creased steadily and in a similar fashion in $Z$ the controls. In PD patients the maximum ratio achieved was lower and started to plateau after about $100 \mathrm{~min}$. This observation $\subseteq$ expresses the diminished capacity of the stri- 0 atum in PD patients to retain tracer (mainly $O$ in the form of fluorinated dopamine and HVA). In patients showing the "on-off" phenomenon, an even further decrease of this capacity was demonstrated. PSP patients showed a comparable impairment of $\Rightarrow$ striatal dopaminergic function but the $\mathrm{HC} \stackrel{\text { of }}{\rightarrow}$ patient had a normal storage capacity. The $\bar{C}$ HD patients showed a variable degree of 흐 diminished tracer uptake.

MORPHOLOGICAL CHANGES IN DENDRITIC PARAMETERS OF MEDIUM SPINY NEURONS OF THE STRIATUM IN PARKINSON'S DISEASE TH McNeill, L Koek, S Brown, RJ Joynt. University of Rochester, Rochester New York, USA

This study examined quantitatively the receptive dendritic profiles of medium spiny neurons of the striatum, the principal targe neuron population for nigrostriatal cells, $t \&$ determine if these neurons undergo compero satory dendritic growth or regression as the. result of the dopaminergic afferent cell losf in Parkinson's disease. Tissue used for these studies was obtained at necropsy from cases clinically and histologically diagnosed to have Parkinson's disease and from agematched normal cases which had no history of neurological or psychiatric illness. Tissues were prepared by the Golgi Cox method according to Van der Loos. Similar to what has been described previously using the Golgi Cox or rapid Golgi methods in normal human neostriatum, medium spiny neurons in aged-matched control brains were characterised by an oval cell body and smooth primary dendrites with increasing spine density on secondary and distal segments. However, medium spiny neurons in Parkinsonian brain were atrophic with severely degenerated dendritic arbors and few dendritic spines. These data suggest that the loss of dopaminergic afferents in Parkinson's disease results in postsynaptic degeneration of medium spiny neuron dendrites and provides a morphological basis for the reduc- $N$ tion in dopaminergic postsynaptic binding sites in the striatum as well as for changes in motor function associated with Parkinson's disease. Supported by AG0354 and 03644. 
SECRETION OF NEURON-SPECIFIC ENOLASE, PROLACTIN, GROWTH HORMONE, LH AND FSH BY PITUITARY TUMOURS IN VITRO

NF Lawton, AJ Evans, JD Pickard, S Perry, B Davies. Wessex Neurological Centre and the Departments of Biochemistry, Surgery and Neuropathology, Southampton University Hospitals, Southampton

Neuron-specific enolase (NSE) is a cell marker for the diffuse neuroendocrine system and is found in both endocrine-active and "functionless" pituitary tumours by immunocytochemical staining. Because of its association with endocrine activity the presence of NSE in tumours which are apparently functionless is of particular interest. We have measured the production rate of NSE in the supernatant from primary explant cultures of 24 adenomas of which 11 were endocrine-active and 13 "functionless". Tumour type was first defined by measurement of plasma hormone levels and hormone secretion rate in vitro. Measurement of prolactin secretion in vitro provided a clear distinction between true prolactinomas and "functionless" tumours causing hyperprolactinaemia by stalk compression (pseudoprolactinomas). Tumours from acromegalic patients secreted the expected large quantities of growth hormone (GH) in vitro. Very low levels of GH secretion by other tumours confirmed absent or minimal contamination of tumour tissue by normal pituitary. "Functionless" tumours as a group secreted significantly greater amounts of LH and FSH in vitro compared with endocrine-active tumours $(p<0.01)$. Plasma levels, however, were usually normal. The secretion rate of NSE by "functionless" tumours was significantly increased compared with endocrine-active tumours $(p<0.01)$.

The results confirm that "functionless" tumours frequently masquerade as prolactinomas and that they secrete modest amounts of gonadotrophins in vitro. A high production rate of NSE is characteristic of the cell type from which a subgroup of "functionless" tumours derive.

OBSERVATIONS DURING TRANSIENT GLOBAL AMNESIA

J Hodges and CD Ward. The Radcliffe Infirmary, Oxford

We recently examined three patients during attacks of transient global amnesia. Two were video recorded and extracts will be shown.
One patient, a 67 year old man, underwent extensive neuropsychological testing during his attack. This confirmed a profound anterograde deficit of verbal and non-verbal memory; which was still moderately defective 12 hours after the episode. Incidental (spatial) memory was relatively preserved. There were substantial savings, without conscious recall or recognition on a repeated cognitive task (object assembly). Short-term memory (digit and block-tapping span) was normal. In tests of remote memory, his recognition of famous faces (110 photographs, spanning six decades) was normal. On a famous events test (spanning five decades) he correctly distinguished real from fictitious events, but his dating of them was markedly impaired. On retesting post-attack, this was normal. Again, there were temporary dating problems on testing personal remote episodic memory (modified Crovitz test). The other two patients appeared to have a very extensive retrograde amnesia, although this was not formally tested.

Anterograde and remote memory can be differentially affected during transient global amnesia. Apparent retrograde amnesia may be due to defective chronological sequencing.

\section{IS NARCOLEPSY-CATAPLEXY AN IMMUNE} DISORDER?

C Lock, N Langdon, K Welsh, A So, J Trowsdale, D Parkes. Tissue Typing, Guy's Hospital, London; Imperial Cancer Research Fund; Department of Neurology, King's College Hospital, London

The MHC region genetic marker HLA DR has been observed in all individuals with classic narcolepsy-cataplexy so far typed in Japan, France and the UK. This is the strongest HLA and disease association known; yet is unexpected because MHC region products are tightly involved in specific immune responses, and narcolepsy-cataplexy has never been considered an immune disorder. Could therefore a gene, not involved in immunity but in close linkage disequilibrium with that coding for $\mathrm{DR}_{2}$, explain the result? Precedents do exist: for example the two genes coding for the enzyme 21-OH map between class II MHC and class III C4A and C4B genes.

To tackle the problem we have, as a preliminary step, isolated DNA from patients with narcolepsy-cataplexy and from tissuetype matched controls, and carried out a Southern blot analysis using a range of restriction enzymes and a set of gene probes which span the class II MHC region. Results indicate that there is a specific class II MHZ sequence which is common to all narcolepsy cataplexy patients. This probably indicates that the disease has a strong immunologicaf component, and we have recently found olie goclonal bands in the cerebrospinal fluid of a narcoleptic patient.

PROXIMAL SLOWING AND CONDUCTION BLOCK IN ACUTE AND CHRONIC DEMYELINATING NEUROPATHY

KR Mills, NMF Murray. National Hosp $\frac{\overline{0}}{\overline{0}}$ tal, Queen Square, London

Percutaneous electrical stimulation over the cervical spine excites the motor roots at theion exit foramina. This technique has been used to investigate ulnar nerve conduction in $2 \%$ patients with acute (within three weeks of onset) Guillain-Barré syndrome (GBS), 1 (థ patients with chronic inflammatory demyे elinating neuropathy, five patients wite paraproteinaemic neuropathy and three pai tients with hereditary sensory and moto neuropathy type I. Results were compare with 25 healthy control nerves. Conduction velocity over three nerve segments (elbow to wrist, axilla to elbow and cord to axilla) wa马 determined and the area of compound niss-cle action potentials evoked in abductof digiti minimi was used to assess conductor block. In patients with acute GBS, abror malities were typically patchy with severa showing evidence of proximal conduction block without associated slowing of conduc tion. In contrast, in chronic demyelinating neuropathy of various aetiologies, the $\mathrm{ab}$ normalities were characterised by slowing of conduction in all nerve segments without ev idence of block. The technique is superior t $\mathcal{Q}$ the measurement of $F$ wave latency in th $\overrightarrow{\bar{F}}$ assessment of proximal conduction since i allows direct measurement of conduction velocity between the cord and axilla and the degree of block can be quantitatively evalus ated.

RETINAL VASCULAR ABNORMALITIES ARE COMMON IN FACIOSCAPULOHUMERAL MUSCULAR DYSTROPHY (FSH)

RB Fitzsimons, EB Gurwin, AC Bird. Na O tional Hospital, Queen Square, and. Moorfields Eye Hospital, London

Coats' syndrome ("non-hereditable" ex udative retinal detachment) has been re ported occasionally in FSH; maculao 
scarring may antedate FSH symptoms*. Since such eye lesions probably result from capillary leakage, we sought to determine by fluorescein angiography whether there was any general association between FSH and retinal vascular disease.

Peripheral retinal telangiectasis (often with capillary closure and fluorescein leakage) was demonstrated in 48 of 65 FSH subjects studied, but not in control spouses. In one FSH family the propositus was treated because of parafoveal oedema associated with posterior pole exudates, and 12 other subjects (including an abortive case in a "skipped" generation) had asymptomatic telangiectasis.

There was no correlation between severity of muscle disease and extent of retinal telangiectasis. Most patients had ophthalmoscopically normal eyes; relevant posterior pole changes were seen in three patients. Exudative retinal detachment and scarring are rare causes of potentially preventible visual loss in FSH, may occur in infancy, and complicate much more frequent underlying capillary abnormalities. Indirect ophthalmoscopy should therefore be performed on infants at genetic risk of FSH.

This study raises the question of whether microvasculature abnormalities are important in the pathogenesis of muscle disease in FSH.

*Joint Moorfields Eye Hospital/
Hammersmith Hospital report.

PATHOGENESIS OF ACUTE ENCEPHALITIS

CR Kennedy, RO Robinson, DAJ Tyrrell, HB Valman, ADB Webster. Department of Paediatrics, Guys Hospital, London and Clinical Research Centre, Harrow, Middlesex

The pathogenesis of the majority of cases of acute encephalitis is unknown. It is assumed that they are virus related. If this is the case, it seems possible either that they are the outcome of unusual neurotropic viruses, or that the host is reacting abnormally to a range of common viruses. Thirty two patients, 29 children and three adults with acute encephalitis, were studied prospectively. By using a wide range of techniques for virus identification as well as measures of their cellular and humeral immunological response, we have attempted to test these two hypotheses. Three patients were found to have an alternative non-infectious cause for their acute encephalopathy. Of the remaining 29 , evidence of recent infection with identified viruses was found in 25 . In 11 of these there was evidence of direct infection of the central nervous system. The remaining 14 had evidence of systemic but not central nervous system infection. The range of viruses identified were those commonly found in the community at large. No immunological deficits or evidence for pathogenic mechanisms were identified. However IgG subclasses I and III were acutely raised in the majority with a further increase in IgG III six months later. The implication of these changes is currently the subject of further investigation. It seems probable therefore that the pathogenesis of acute encephalitis lies less in the nature of the infective agent but more in the nature of the host response.

MEDULLARY CONTROL OF SYSTEMIC BLOOD PRESSURE: A PHYSIOLOGICAL, BIOCHEMICAL, AND NEUROENDOCRINOLOGICAL

CHARACTERIZATION OF THE VEGETATIVE LEVEL OF THE CENTRAL NERVOUS SYSTEM

JP Conomy, KL Barnes, CM Ferrario. The Cleveland Clinic Foundation, Cleveland, Ohio, USA

Among Jacksonian hierarchies of nervous system organisation, the most fundamental is the vegetative. Its operations are automatic, unwilled and insentiate. Yet its functions are essential to the integrity of the organism and its principles of operation similar to those levels concerned with higher functions. In hierarchical conceptualisation Jackson may well have laid the groundwork for modern concepts in neuroendocrinology and cardiovascular neurobiology. Insights into the central nervous system regulation of vegetative function have been provided by investigations from the group of medullary centres concerned with cardiovascular control. Specifically a neuronal system surrounding the fourth ventricle of the dog has been shown to influence arterial blood pressure. Stimulation of the canine area postrema increases blood pressure and peripheral resistance, while ablation leads to mild hypotension and reduced haemodynamic variability. In contrast, stimulation of the nucleus of the tractus solitarius causes an abrupt bradycardia associated with systemic hypotension and ablation of this central baroreflex relay leads to hypertension with increased circulating vasopressin. The input and output connections of these two medullary centres are widely distributed in cardiopulmonary system receptors and the brain. The system is both angiotensinergic and catecholaminergic. $\mathbf{R e}-$ cent evidence suggests that the pressor actions of angiotensin exerted through the area postrema in the dog may be vasopressindependent, that integrity of this region may be necessary to produce experimental renal hypertension and that endogenous opioids participate in cerebral cardiovascular regulation. Thus the counterbalancing, interdependent mechanisms of cardiovascular regulation mediated through the area postrema and nucleus of the tractus solitarius both provide an excellent example of Jacksonian vegetative function, and are now being shown to operate via the most recently discovered principles of neurohormonal and neuropeptide interactions.

THE RESULTS OF REMOVAL OF TEMPORAL LOBE NEOCORTEX IN THE TREATMENT OF EPILEPSY IN 40 PATIENTS

A Coughlan, M Farrell, O Hardiman, BO Moore, H Staunton. The Richmond Institute for Neurology and Neurosurgery, Dublin

A temporal lobectomy was performed in forty patients with intractable epilepsy. Ino the operation neocortex only was removed leaving the limbic structures amygdala ando hippocampus intact. The patients suffereds from a variety of simple partial, complexo partial and secondary generalised seizures? The follow-up period has ranged from two months to ten years.

Using a slightly modified version of the Crandall surgical therapeutic group classification, $58 \%$ became seizure free. A further $25 \%$ showed a worthwhile improvement. Seventeen percent showed no worthwhile improvement. In all cases the approach to lateralisation and localisation was based entirely on the interictal spike, in many cases elicited for the first time by intravenous methohexitone. In all cases except one, a spike was recorded with surface recordings at surgery, usually elicited by methohexitone. We are currently making depth recordings from amygdala and hippocampus at surgery, but leaving these structures intact even when spikes are recorded.

The pathology was studied in all cases. The changes included mainly neuronal ectopia and disturbed neuronal architecture, but also Chaslin's gliosis. The significance of these changes is as yet uncertain.

It may be that the temporal neocortex is required in many instances for the conversion of a deeper epileptogenic spike (paroxysmal depolarisation shift) into a seizure. 
MULTI-CENTER VALIDATION STUDY OF ULTRASOUND FOR QUANTIFICATION OF CAROTID ARTERY DISEASE

JF Toole, D O'Leary, J Ricotta, B Goldberg, J Raines. Bowman Gray School of Medicine, Winston-Salem, NC. USA

Patients ( $\mathrm{n}=1211,2422$ carotid arteries) were entered into a longitudinal study to assess the sensitivity/specificity, reliability/ validity, and accuracy of real-time ultrasound compared with arteriography and pathology, as well as progression/regression of plaque. Results indicate that B-scan and arteriography are comparable. The coefficient of variation for lesion width was $32 \%$ by B-scan and $33 \%$ by arteriography. For residual lumen, it was $30 \%$ for B-scan and $25 \%$ for arteriography.

The implications of these results for population based assessment of prevalence of pre-clinical atherosclerosis and for epidemiologic studies are discussed.

MULTIPLE SCLEROSIS IN THE NORTH-EAST OF SCOTLAND: A NEW GENETIC ASSOCIATION WITH HLA-DQwl

DA Francis, AW Downie, JEC Hern, JR Batchelor, WI McDonald. Department of Neurology, Aberdeen Royal Infirmary, Aberdeen

We have studied the influence of HLA antigens on the susceptibility to multiple sclerosis (MS) and subsequent clinical course in 200 patients from the Grampian Region of Scotland, an area of high disease prevalence. The frequency of HLA DR2 in affected individuals was similar to other northern European patient groups but was also high in 130 local controls $(49 \%$ vs $41 \%, p=N S)$. The increased frequency of DR2 in the normal Grampian population was significant when compared with other European control populations $(p<0.001)$. A significant association was demonstrated between MS and HLA DQwl $(77 \%$ vs $62 \%),(p=0.006)$ even though this antigen was also of increased frequency in controls.

DRw6 was significantly increased and DR4 significantly decreased in patients with severe disease (K.D.S. > 5.0) within 5 years $(p<0.005)$ suggesting some genetic heterogeneity between different clinical groups of patients, but neither HLA DR2 nor DQwl appeared to influence disease progression.

This study raises the possibility that the high prevalence of MS in the region may partly be related to the over-representation of DR2, or a closely linked antigen, in the local population. Given the recently de- scribed close linkage between HLA DR2 and DQwl, our findings suggest that DQwl is a potential candidate for the MS susceptibility gene.

ASSESSMENT OF CEREBRAL HAEMODYNAMIC RESERVE: CORRELATION BETWEEN PET AND $\mathrm{CO}_{2}$ REACTIVITY MEASURED WITH THE ${ }^{133}$ XENON INJECTION TECHNIQUE

S Herold, MM Brown, RSJ Frackowiak, J Marshall, AO Mansfield, DJ Thomas, T Jones. MRC Cyclotron Unit, Hammersmith Hospital, London

Although the combined measurement of cerebral blood flow (CBF), blood volume (CBV) and oxygen extraction (OER) by positron emission tomography (PET) provides information about haemodynamic reserve and oxygen carriage reserve in carotid occlusive disease, more readily available techniques are needed for assessing larger numbers of patients. The state of compensatory vasodilatation which can be demonstrated as high $\mathrm{CBV}$ and low $\mathrm{CBF} / \mathrm{CBV}$ ratio with PET may also be detected as a reduced CBF-response to hypercarbia. CBF alone can be measured by single photon techniques.

For comparison, PET studies and measurements of $\mathrm{CO}_{2}$-reactivity (using the ${ }^{133} \mathrm{Xe}$ injection technique) were performed in 21 patients; 19 had uni- or bilateral carotid artery occlusion, two had high grade stenosis.

For the symptomatic hemispheres a significant correlation was found between the $\mathrm{CBF} / \mathrm{CBV}$ ratio and $\mathrm{CO}_{2}$-reactivity. The relation between $\mathrm{OER}$ and $\mathrm{CO}_{2}$-reactivity showed a similar threshold effect as observed for OER and CBF/CBV ratio. Of 6 patients with $\mathrm{CO}_{2}$-reactivities below $1.5 \%$, five had raised OERs, whereas in the remaining patients with reactivities above $1 \cdot 5 \%$, OER was normal in all cases.

It is concluded that by measuring $\mathrm{CO}_{2}$-reactivity, patients who have started to call upon oxygen carriage reserve and are therefore most compromised can be reliably identified.

A DOUBLE-BLIND CONTROLLED TRIAL OF HIGH-DOSE INTRAVENOUS METHYL PREDNISOLONE IN THE TREATMENT OF MULTIPLE SCLEROSIS NM Milligan, PJ Hughes, DAS Compston. University Hospital of Wales, Cardiff

A randomised double-blind controlled trial of high-dose intravenous methyl predn $\bar{b}$ solone was carried out in 51 individuals wit multiple sclerosis; 22 patients were in acue relapse and 29 had chronic progressive disease. After a base-line assessment using the Kurtzke clinical scales for disability visual auditory and somatosensory evoke potentials, CSF examination for cell cou IgG and complement (C9) concentrations and enumeration of peripheral blood lym. phocyte sub-populations, each patient wa霂 randomly allocated to intravenous treat ment with methyl prednisolone $(500 \mathrm{mgs})$ a saline placebo as a single daily dose for fie days. At completion of treatment, clinic and peripheral blood measurements we repeated; all clinical and laboratory meajs surements were reassessed at four weeks. क

In patients with acute relapse, there was $\vec{\theta}$ significant decrease in disability scores at one and four weeks in the methyl prednisolono treated group compared with controls ( $p$. 0.01 ). In the chronic progressive group, dis ability scores at 4 weeks were alșo significantly lower after treatment wite methyl prednisolone $(\mathrm{p}<0.05)$, largely dịe to improvement in pyramidal function. Laboratory studies showed that periphera blood $\mathrm{T} 8+$ cells returned into the normal range, CSF cell count and IgG concentration fell, whereas $\mathrm{C} 9$ was increased \&fter active treatment; evoked potential lategcies remained unchanged.

LABORATORY INDICES OF DISEASE ACTIVITY MULTIPLE SCLEROSIS

AJ Thompson, J Brazil, C Feighery, Eब Martin, M Hutchinson. St Vincents' Hos tal, St James' Hospital, Dublin

There is a need for an objective laborato marker for the assessment of disease activiry in multiple sclerosis (MS). Three possib laboratory indices, CSF myelin basic protein (MBP), peripheral blood $\mathrm{T}$ lymphocte counts and CSF IgG abnormalities we simultaneously examined in 15 patients with clinically definite MS. The frequency of lapses and the development of disability were used as clinical estimates of disease activity.

A significant association between the of tection of MBP in CSF and relapse was dem onstrated $(p<0.01)$. In contrast ser21 estimations of peripheral blood $\mathrm{T}$ lymph $\overline{\overline{\mathrm{T}}}$ cyte numbers showed no consistent lationship between reduced suppressor $N$ cells and relapse. Persistently reduced cells, including both helper and suppresset subsets were however associated with disease 
activity as measured by the development of disability. The detection of MBP in CSF during remission was also associated with the development of disability and there was a significant correlation between the detection of MBP in the CSF and reduced suppressor $T$ cell counts in the peripheral blood $(p<0.05)$. No association was found between the CSF IgG index or oligoclonal banding and clinical or other laboratory markers of disease activity.

Thus both the detection of MBP in CSF during remission and persistently reduced peripheral blood $\mathrm{T}$ lymphocytes were associated with the development of disability during the course of this one year study.

MULTIPLE SCLEROSIS GLYCOLIPIDS AND VIRUS INDUCED AUTO-IMMUNITY

HE Webb, Rayne Institute, St Thomas' Hospital, London

Multiple sclerosis is thought by many to be the long term result of a virus infection acquired in childhood in susceptible HLA types. Some studies suggest that an immune reaction occurs directed against CNS myelin components. Most work has been on the immunogenicity of protein components of viruses and cell membranes and has been unrewarding. Little attention has been paid to glycolipids as they were erroneously thought to be too non-immunogenic. Enveloped viruses (measles, mumps, parainfluenza, herpes) which may be associated with MS, take host cell glycolipid into their new envelopes during budding from the host cell after replication. Glycolipid presented by a virus in this way is antigenic and could produce an anti-glycolipid auto-immune disease within the CNS. Using an RNA budding virus causing focal $\mathrm{T}$ cell mediated demyelination of the CNS in mice we have shown that animals infected develop antiglycolipid anti-body and have labelled the brain derived envelope virus with antiglycolipid sera. These facts are discussed in relation to anti-glycolipid activity found in MS patients and the production of experimental demyelination in animals using glycolipids rather than myelin basic protein.

MAGNETIC RESONANCE IMAGING IN SYRINGOMYELIA - A COMPARATIVE STUDY WITH CT MYELOGRAPHY

JE Gillespie, RA Metcalfe, JPR Jenkins, P Butler, I Isherwood. Departments of Diagnostic Radiology and Neurology, University of Manchester
The aim of the study was to compare magnetic resonance imaging (MRI) with CT myelography in the diagnosis of cranio-cervical anomalies in patients with well-defined neurological deficit. MRI has significant advantages over current imaging methods in the assessment of the cranio-cervical region and spinal cord in that it is non-invasive, uses non-ionising radiation, images directly in the sagittal plane and does not require the use of subarachnoid contrast medium.

In 30 patients aged between 17 and 75 years cervical myelography was followed three to five hours later by CT myelography of the lower posterior fossa and upper spinal canal. MRI was performed prior to or within 24 hours of the CT myelogram. $T_{1}$ and $T_{2}$ weighted Spin Echo sequences were used in the mid-line sagittal plane to outline the brain stem and cord. The extent of the lesion was defined.

CT myelography demonstrated 10 intramedullary cavities. MRI showed eight of these and more clearly defined their extent and associated tonsillar ectopia. However, in two cases of localised cavitation within a normal size cord, MRI did not show any abnormality. These reasons for this will be discussed.

MR IMAGING (MRI) IN ISOLATED

NEUROLOGICAL LESIONS

IEC Ormerod, P Rudge, WI McDonald, G Johnson, EGPH du Boulay, AM Halliday, BE Kendall, IF Moseley, D MacManus. Institute of Neurology \& National Hospital, Queen Square, London

MRI readily detects lesions in multiple sclerosis (MS). Since optic neuritis and acute brainstem lesions are common initial manifestations of MS we were interested in determining the frequency with which multiple lesions could be detected in patients with these conditions. We used a $0.5 \mathrm{~T}$ Picker superconducting MRI system, to compare the findings with evoked potential studies in the same patients and with MRI from 116 patients with clinically definite MS (aged 15 to 74 years, mean 39.9 years) and 50 control subjects (aged 18 to 60 years, mean 24 years). Of 38 adults with isolated optic neuritis, aged 16 to 48 years (mean $32 \cdot 2$ years), 24 had abnormalities on the MR scan. Only 13 patients had evoked potential abnormalities indicating lesions outside the visual system. Twenty-seven patients aged 21 to 49 years (mean 31.5 years) with symptoms and signs of an isolated brainstem lesion were studied. In 25 , a lesion within the brainstem was visualised and in 20 there were additional clinically silent abnormalities in the supratentorial scans. MRI was more sensitive than evoked potentials in detecting brainstem and other lesions except in the optic nerve. The prognostic significance of multiple lesions in monosymptomatic patients at presentation remains to be determined. it is not yet possible to make a diagnosis of MS on MRI alone.

HEARING LOSS IN STREPTOCOCCAL MENINGITIS RLC Kay, TJL Alexander. Guy's Hospital London and Cambridge Veterinary School

Hearing loss as a complication of bacterial meningitis is well recognised although its pathological basis remains uncertain.

An experimental model of group D streptococcal meningitis was studied in guinea pigs. Hearing was assessed by brainstem auditory evoked potentials (BAEP), and histological examination of brain and temporal bone was performed. Cochlear sepsis was invariably present in cases of meningitis in which the BAEP threshold was significantly elevated $(>30 \mathrm{~dB})$, but absent in those with normal thresholds. Inflammatory cells were seen in the perilymphatic space, and in the cochlear aqueduct which connects the former to the subarachnoid space. In con? trast, the endolymphatic space was generall free from infection.

We have shown that BAEP audiometry can be used to demonstrate cochlear function in bacterial meningitis. It is suggested that cochlear rather than eighth nerve involvement is primarily responsible for the hearing loss complicating meningitis. The conduit for spread of infection from meninges to the cochlea appears more likely to be the cochlear aqueduct than the internal auditory canal, which has no direct communication with the perilymphatic space.

RELATIONSHIP BETWEEN THE SUBARACHNOID AND PERIVASCULAR SPACES IN THE HUMAN NER VOUS SYSTEM

RO Weller, M Hutchings. University Medical School, Southampton

For many years it has been assumed that there is direct continuity between the subarachnoid and perivascular spaces in the human brain. This concept is re-examined in the present study. The relationships of the arachnoid and pia mater to the entry of blood vessels into the cerebral cortex were examined by scanning electron microscopy 
(SEM) and by light microscopy in normal brains and in brains following subarachnoid haemorrhage and leptomeningitis. SEM showed that the pia mater was reflected on to the vessels of the subarachnoid space and did not accompany vessels into the brain as previously reported. Thus the subarachnoid space does not connect freely with the perivascular spaces and blood from subarachnoid haemorrhage does not pass readily into the perivascular spaces. In meningitis, inflammatory cells expand the perivascular and subpial spaces; these compartments are in continuity and separated from the subarachnoid space by the pia mater. The implications of this anatomical arrangement of the leptomeninges at the surface of the brain will be discussed.

NEW TECHNIQUE FOR THE

NEUROPHYSIOLOGICAL ASSESSMENT OF SMALL FIBRE NEUROPATHY: RESULTS IN 25 PATIENTS
GA Jamal, AI Weir, S Hansen, JP Ballantyne. Institute of Neurological Sciences, Glasgow

Conventional nerve conduction (NC) and EMG studies are of little value in the assessment of neuropathies which mainly or exclusively involve the small nerve fibre population. By the measurement of thermal thresholds, the technique of Jamal et al. ${ }^{12}$ provides a reproducible index of function in the small fibre pathways, in particular the AS and $C$ group.

The results of the application of that technique to a group of 25 patients with clinically suspected small fibre neuropathy are presented. The method recorded an abnormality in $100 \%$ of the patients when conventional NC and EMG and vibration threshold ${ }^{3}$ studies were normal. The abnormalities were more frequent and more severe at the ankles than at the wrists. This technique provides, for the first time, an accurate, easily performed and reproducible laboratory investigation of clinically suz pected small fibre neuropathy.

\section{References}

${ }^{1}$ Jamal GA, Hansen S, Weir AI, Ballantyne J $\overrightarrow{\mathrm{F}}$ An improved automated method for the meat surement of thermal thresholds. 1. Norm subjects. J Neurol Neurosurg Psychiat 1985;48:354-60.

2 Jamal GA, Hansen S, Weir AI, Ballantyne J An improved automated method for the meQ surement of thermal thresholds. 2. Patien with peripheral neuropathy. J Neurol Neurosurg Psychiatry 1985;48:361-6.

${ }^{3}$ Goldberg JM, Lindblom U. Standardised method of determining vibratory perceptiob thresholds for diagnosis and screening in neథ rological investigation. $J$ Neurol Neurosurg Psychiatry 1979;42:793-803. 